\title{
Resilient Market Timing Strategies For Global Equities
}

\author{
Heng-Hsing Hsieh, PhD, CFA, University of the Western Cape, South Africa \\ Kathleen Hodnett, PhD, University of the Western Cape, South Africa \\ Paul van Rensburg, PhD, University of Cape Town, South Africa
}

\begin{abstract}
The systemic impact of the global financial crisis of 2008 reveals that there are periods of uncertainty during which most asset classes experience substantial drawdown, rendering diversification an ineffective risk management tool. The desired exposures to risky assets such as stocks, bonds and commodities during these periods of systemic risk should be zero. This paper tests the effectiveness of two market timing strategies that intend to provide early signals for portfolio protection during turbulent times: a filter rule strategy based on the portfolio drawdown $(D D)$ and drawup (DU) thresholds; and an exponential moving average (EMA) strategy based on the crossover of the fast moving average (FMA) and the slow moving average (SMA) of the fund values. The pre-specified market timing strategies are tested on the total return index of the Morgan Stanley Capital International World (MSCI World) Index since the inception of the index in 1997 through 2008. Both the optimal filter rule strategy and the optimal EMA strategy achieve Sharpe ratios that are higher than the Sharpe ratio of the unprotected MSCI World Index. The comparison of the historical risk-return characteristics reveals that the timing of protection is more accurate for the EMA strategy. As is the case for all market timing strategies, the signals provided by the protection mechanism lag the actual economic events. Thus, the market timing strategies tend to be more effective for prolonged economic downturn.
\end{abstract}

Keywords: Market Timing; Drawdown; Systemic Risk; Moving Average; Global Financial Crisis; Risk Management; Active and Passive Portfolio Management

\section{RESEARCH BACKGROUND}

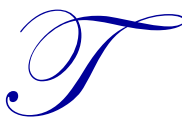

he global financial crisis of 2008 impacted on all asset classes, rendering diversification an ineffective mechanism for controlling downside risk. Empirical research conducted by Billio, Getmansky and Pelizzon (2009) indicates that hedge funds that target at generating absolute returns with low levels of systematic risk have failed to cope with the global financial crisis. During the abnormal state of the economy, the benefits of diversification dissipate as many insignificantly-correlated asset classes experience significant drawdowns simultaneously (Faber, 2009). Faber (2009) examines the maximum drawdown of the following asset classes, namely U.S. stocks, EAFE stocks, commodities and the real estate investment trusts (REIT) over the period from 1973 to 2008 . The results reveal that all asset classes have drawdown around $40 \%$ to $60 \%$ with the exception of the U.S. government bonds, which declines almost $20 \%$ over the examination period. The buy-andhold investors find themselves unable to recover from the severe drawdown due to their limited life span and personal liquidity constraints. Faber (2009: 3) regards any reason to hold any asset during a global financial crisis a "decidedly unwise course of action".

Hedge fund strategies that claimed to generate absolute returns throughout the business cycle also suffered from the impact of the global financial crisis. Ballio, Getmansky and Pelizzon (2006) analyze the time-varying exposures of hedge fund indexes to risk factors using switching regime beta modeling over the period from 1994 to 2005. They find that hedge fund exposures to systematic risk factors are very low during the normal state of the market. However, the exposures increase drastically when the market is characterised by negative S\&P 500 returns and high volatility. Calson and Steinman (2008) examine the relationship between financial market conditions and the likelihood of U.S. hedge fund failures over the period from 1994 to 2006. They find that hedge funds are more 
likely to fail during the periods when there is a four standard deviation drop in the S\&P 500 Index as well as a four standard deviation fall in the value of the U.S. dollar.

Further investigation on the effects of financial crises on hedge fund exposures conducted by Billio et al (2009) indicate that although the hedge fund exposures to the S\&P 500 returns decline or turn negative during crises, the average volatility and correlation of hedge fund returns increase during global financial crises. The study results of Billio et al (2009) also suggest that the increased volatility and correlation between hedge fund returns are due to the increase in their common exposures to latent risk factors that are potentially related to margin spirals, massive redemptions, credit freezes, and interconnectedness between financial institutions during financial crises. These findings raise the question as to whether the hedge fund strategies are indeed "better hedged" than traditional mutual funds and asset classes during global financial crises.

Given the inability to diversify the systemic risk of global financial crises, market timing strategies that seek to shift capital between risky assets and cash, based on the expected market trends, can potentially be more resilient than a traditional buy-and-hold strategy. Perfect market timing is defined by Bodie, Kane and Marcus (2008) as "the ability to tell (with certainty) at the beginning of each year whether the $S \& P 500$ portfolio will outperform the strategy of rolling over 1-month T-bills throughout the year". In other words, a perfect market timing strategy will "hold common stocks during bull markets and cash equivalents during bear markets" (Sharpe, 1975: $60)$.

This paper undertakes to develop market timing strategies that trigger protection against significant drawdown in turbulent times by reducing equity investments or creating a synthetic cash position using derivative overlay. The market timing strategies developed in this research include a filter rule strategy that triggers and releases protection based on the drawdown (DD) and the drawup (DU) estimated from the most recent peak and trough, and an exponential moving average (EMA) strategy that triggers and releases protection based on the crossover of the fast moving average (FMA) and the slow moving average (SMA). These two strategies are tested on the Morgan Stanley Capital International World (MSCI World) Index since its inception from 1 January 1970 to 31 December 2008. The ultimate goal of this research is to develop successful market timing strategies that preserve the value of the MSCI Index during significant drawdown, without introducing significant drag in its upside profit potential when the market rebounds.

\section{LITERATURE REVIEW}

Early research regarding market timing generally focuses on the potential gains and the required accuracy of market timing strategies. Inaccurate market timing entails the exposure to larger losses with unrecoverable transaction costs incurred when errors are made (Sharpe, 1975). Taking into account the probability of imperfect timing, Sharpe (1975) conducts a study to answer the question: "how superior must one's predictions be to implement a market timing style effectively?" by comparing the returns on three strategies, namely a buy-and-hold strategy, a perfect market timing strategy and a cash equivalent strategy over the period from 1929 through 1972. The test results suggest that the gains from successful market timing strategies are modest at best (less than $4 \%$ per year), and the gains are only earned by managers who can predict correct timing with considerable accuracy (more than $70 \%$ of the time).

Chua, Woodward and To (1987) view a buy-and-hold strategy as a strategy that has $100 \%$ accuracy in forecasting the bull market and 0\% accuracy in forecasting the bear market. Chua et al (1987) investigate the potential gains from market timing in Canada over the period from 1950 to 1983. Using a series of Monte Carlo simulations to resemble estimates of the gains from less-than-perfect forecasts, they find that successful market timing strategies require a minimum accuracy of $80 \%$ in forecasting the bull and bear markets. However, their results also show that less-than-perfect timing strategies increase the investor's chance of avoiding a bear market at the expense of lowering the profits earned in the bull markets.

Contrary to the findings of Sharpe (1975) and Chua et al (1987), Clarke, FitzGerald, Berent and Statman (1989) argue that prior studies find little fruit in market timing by assuming investors have zero access to information regarding forecasting market trends. They further argue that even investors with just modest information regarding future market trends have potential to generate substantial gains. In their study, a linear model with 
changes in GNP as its only input is found to generate $5.9 \%$ annual return in excess of the return earned by a buyand-hold strategy with an R-squared of only $9 \%$.

The success of a market timing strategy is found to be firm size dependent. Kester (1990) conducts a study on the potential gains and required predictability of market timing with small versus large caps over the period from 1934 to 1988. Test results indicate that switching between small caps and cash are more profitable and requires less prediction accuracy compared to switching between large caps and cash. The success of market timing is also found to be time period specific. Bauer and Dahlquist (2001) evaluate monthly, quarterly and annual market timing strategies over the sub-periods from 1926 through 1999. Their results reveal that market timing strategies are less likely to be successful in major bull markets, such as the period from 1995 to 1999.

More recent studies on market timing focus on the developing trend-following models to profit from effective switching between asset classes. Resnick and Shoesmith (2002) adopt the probit model to forecast economic recessions using the yield curve spread between the 10-year Treasury yield and the 3-month Treasury yield as the model input over the period from 1971 to 1999 . The simulated out-of-sample results indicate that a market timer, who is able to switch between stocks (bonds) a month before a bear market (a bull market), at a 50\% probability, could have earned an annual return of $2.29 \%$ in excess of a buy-and-hold strategy. Ang and Bekaert (2004) suggest that global equity returns exhibit unusually high correlations during economic turmoil. Thus, during periods of high persistent uncertainty, a model that signals investors to switch primarily to cash could have added substantial value. Based on this argument, Ang and Bekaert (2004) devise a regime-switching strategy based on the observed regimes with different correlations and expected returns. When this model is tested on a universe of developed equity markets over the period from 1975 through 2000, it is found that the regime-switching strategy outperforms a buy-and-hold strategy in the out-of-sample period from 1985 through 2000.

Faber (2009) tests the ability of a moving average timing model to implement overlay hedging that converts fund exposures to a synthetic cash position during crises. The timing strategy is applied to the 5 common asset class indexes, namely the S\&P 500 Index, the MSCI EAFE Index, the Goldman Sachs Commodity Index (GSCI), the National Association of Real Estate Investment Trusts (NAREIT) Index and the 10-year U.S. Treasury Bond Index over the period from 1900 to 2008. The trading system signals a long position in the index when the monthly index level is higher than its 200-day moving average and vice versa. The results reveal that the timing strategies have few occurrences of both large gains and large losses, with correspondingly higher occurrences of small gains and losses over the examination period. The average returns of the timing strategies are in line with their respective indexes. However, the timing strategies have considerably lower volatilities and drawdown compared to the long-only indexes. Faber (2009) concludes that the moving average trading system is an effective mechanism to avoid the impact of financial market disasters in that it achieves equity-like returns with bond-like volatility and drawdown.

The savings resulted from avoiding the bear market could be more lucrative than the opportunity cost of missing the bull market. This argument is provided by Shilling (1992), who finds that being out of stocks during market downturns outweighs the gains generated by fully investing in stocks during bull markets over the period from 1946 through 1991. Estrada (2008) uses the "black swan" argument of Taleb (2007) to demonstrate the importance of market timing. A black swan is defined by Taleb (2007) as an (outlier) event that has extreme impact, yet cannot be predicted based on past experiences. Estrada (2008) examines the impacts of extreme outliers on longterm portfolio performance, based on the histories of 15 stock market indexes since index inceptions through 2006. Test results indicate that missing the best 10 days would result in the portfolio being $50.8 \%$ less valuable compared to a buy-and-hold strategy, on average. On the other hand, a market timer who avoids the worst 10 days would result in the portfolio being $150.4 \%$ more valuable compared to a buy-and-hold strategy, on average.

In summary, empirical studies suggest that market timing strategies that focus on predicting each bull or bear market requires substantially high probability of success to outperform a passive buy-and-hold strategy meaningfully. However, the gains from market timing can be improved substantially using modest information regarding future market movements to catch on major bull markets or avoid major bear markets. The argument of a black swan seems to support market timing strategies that focus on avoiding major financial market crises, which results in massive savings at an expense of missing some gains of the bull markets. 


\section{DATA AND METHODOLOGY}

The empirical findings on the success of market timing strategies suggest that the primary objective of a practical market timing strategy should be a protection-based strategy that aims at preserving portfolio values during major economic shocks. Taking into account the trading costs involved in switching between asset classes, frequent short-term forecasts are ineffective in that substantially high forecasting accuracy is required to provide modest value, in addition to what is offered by a passive buy-and-hold strategy. Empirical evidence also suggests that the substantial savings available from avoiding major economic shocks outweigh the gains provided by participating in the bull markets. Thus, we focus on developing protection-based market timing strategies that are resilient during major market downturns, such as the oil crisis of 1973, the crash of the dot-com bubble of 2001 and the global financial market crisis in the late 2008.

Two protection-based market timing strategies are developed in this research: a filter rule strategy that triggers and releases protection signals based on the drawdown (DD) and drawup (DU) of the fund value; and an exponential moving average (EMA) strategy that triggers and releases protection signals based on the crossover of the fast moving average (FMA) and the slow moving average (SMA) of the fund value. Both strategies are tested on the MSCI World Index since its inception in 1990 through 2008. The major shocks that affect the world economy during this period include the oil crisis of 1973, black Monday of 1987, the Asian financial crisis of 1997, the Argentinean crisis of 2001, the bursting of the dot-com bubble in 2001 and the subprime crisis of 2007 that led to the global stock market crash in October 2008.

The implementation of the protection-based market timing strategies is based on the lagged 1-month signal generated by the respective strategies. Both strategies start by investing $100 \%$ of their capital in the MSCI World Index until a warning signal is triggered, which converts the entire equity exposure into cash. When the warning signal is released, the strategies resume $100 \%$ of their exposures in the MSCI World Index. The total U.S. dollar return index values for the MSCI World Index since its inception in 1970 is downloaded from the database of DataStream International. The cash returns are proxied by the yield on the U.S. 3-month Treasury bill. A transaction cost of $1 \%$ is assumed for each switch between the MSCI World Index and cash. The performances of the protected strategies and the unprotected index are evaluated based on the annualized geometric return, standard deviation, Sharpe ratio, 5 percent value-at-risk (VaR) and the maximum DD over the examination period.

\section{The Filter Rule Strategy}

The DD of the MSCI World Index in month $t\left(D D_{t}\right)$ is the index's cumulative loss to date (month $t$ ) since the most recent peak of the index's cumulative value as shown in Equation 1. On the other hand, the DU of the MSCI World Index in month $t\left(D U_{t}\right)$ is the index's cumulative return to date since the most recent trough of the index's cumulative value as shown in Equation 2.

$$
\begin{aligned}
& D D_{t}=\frac{\text { Index }_{t}}{\text { Index }_{t}^{\text {max }}}-1 \\
& D U_{t}=\frac{\text { Index }_{t}}{\text { Index }_{t}^{\text {min }}}-1
\end{aligned}
$$

Where:

Index $_{t} \quad$ is the cumulative total return of the MSCI World Index since inception in month $t$;

Index $_{t}^{\max }$ is the maximum value of the cumulative total return of the MSCI World Index since inception to date (that is, month $t$ ); and

Index $_{t}^{\text {min }}$ is the minimum value of the cumulative total return of the MSCI World Index since inception to date (that is, month $t$ ). 
To apply the filter rule strategy on the MSCI World Index, the DD and DU of the MSCI World Index are computed monthly to determine whether protection is required in each month of the examination period. A maximum tolerable DD filter is first set to represent the maximum level of tolerance within which the protection will not be triggered. When the maximum tolerable DD filter is exceeded, a warning signal is triggered to convert the exposure in the MSCI World Index into cash. The fund will stay in cash until the minimum required DU filter is exceeded. The permutations of the maximum tolerable DD filter that triggers cash protection and the minimum required DU filter that lifts the cash protection are simulated from $0 \%$ to $50 \%$, at a $5 \%$ interval. The permutations of DD and DU of the MSCI World Index over the examination period are evaluated based on the annualized geometric return, standard deviation, Sharpe ratio, 5 percent value-at-risk $(\mathrm{VaR})$ and the maximum DD over the examination periods. The best permutation of DD and DU are extracted to enable comparison between the historical performance of the protected index and that of the unprotected index.

\section{The Exponential Moving Average (EMA) Strategy}

The EMA strategy generates signals based on the crossover of the FMA and the SMA computed from the cumulative U.S. dollar total return of the MSCI World Index since its inception in 1970. The FMA and SMA are EMA series that allocate greater weights to recent observations by reducing the weight for each of the older observations exponentially. The FMA and SMA of the MSCI World Index in month $t$ are computed using Equation 3 and Equation 4 respectively:

$$
\begin{aligned}
& F M A_{t}=x \% \times \text { Index }_{t}+(1-x \%) \times F M A_{t-1} \\
& S M A_{t}=y \% \times \text { Index }_{t}+(1-y \%) \times S M A_{t-1}
\end{aligned}
$$

Where:

Index $_{t}$ is the cumulative total return of the MSCI World Index in month $t$;

$x \% \quad$ represents the speed at which the FMA tracks the current value of the MSCI World Index; and

$y \%$ represents the speed at which the SMA tracks the current value of the MSCI World Index.

The FMA in month $t$ allocates $x \%$ of the value to the current MSCI World Index value in month $t$ and (1$x) \%$ of the value to the FMA value in month $t-1$. On the other hand, the SMA in month $t$ is calculated as $y \%$ of the current MSCI World Index value plus (1-y)\% of the SMA value in month $t-1$. Thus, the $x \%$ and $y \%$ represent the speed at which the FMA and SMA track the current value of the underlying index. For the FMA to represent a "faster" moving average series relative to the SMA, the weight allocation of the FMA to the current MSCI World Index value $(x \%)$ has to be greater than the weight allocation of the SMA to the current MSCI World Index value $(y \%)$. When the FMA breaks through the SMA from above, the warning signal is triggered and the exposure in the MSCI World Index is converted to cash in the coming month until the FMA breaks through the SMA from below. The permutations of the rates at which the FMA and the SMA track the MSCI World Index are simulated from 0\% to $100 \%$, at a $10 \%$ interval, over the examination period. The permutations of $x \%$ and $y \%$ for the EMA strategy over the examination period are extracted with the their performances evaluated based on the annualized geometric return, standard deviation, Sharpe ratio, 5 percent $\mathrm{VaR}$ and the maximum drawdown over the examination period. The best permutation of $x \%$ and $y \%$ are extracted to enable comparison between the historical performance of the protected index and that of the unprotected index.

\section{EMPIRICAL FINDINGS}

The results of the permutations for the filter rule strategy and the EMA strategy are first analyzed in this section to define the respective market timing strategies that maximize the Sharpe ratio of the MSCI World Index being protected. The periodic cumulative returns and drawdowns for the protected MSCI World Index and the unprotected MSCI World Index are examined in conjunction with their relative strength over time to identify circumstances under which the market timing strategies are economically beneficial. 
The summarized performance statistics such as the annualized geometric return, standard deviation, Sharpe ratio, 5 percent $\mathrm{VaR}$ and the maximum $\mathrm{DD}$, over the examination period, for the unprotected and the protected indexes are provided in this section. In order to compare and contrast the effectiveness of different levels of protection applied to the MSCI World Index, the performance statistics for a strategy that switches only $50 \%$ of its investment to cash when the protection signal is received (that is, a partially-hedged strategy) are also provided.

\section{Optimal Permutations for the Market Timing Strategies}

The Sharpe ratios for the filter rule strategy based on the simulated permutations of the maximum tolerable DD filter and the minimum required DU filter are demonstrated in the form of a heat map in Table 1. The optimal permutation that maximizes the Sharpe ratio of the filter rule strategy is indicated in the bold box. Examining the heat map in Table 1 reveals that the Sharpe ratio of the protected MSCI World Index is maximized at 38.26\% when the maximum tolerable DD filter and the minimum required DU filter are set at $-5 \%$ and $5 \%$ respectively. The maximum DD for the MSCI World Index over the examination period is $-40.12 \%$. Thus, the permutations that involve DD of more than $40 \%$ would have triggered no protection signals during the period. The Sharpe ratio derived from these permutations is equal to the Sharpe ratio of the unprotected MSCI World Index of 24.30\%.

Practical DD and DU should be in-between the absolute values of $5 \%$ and $20 \%$. Having cash protection triggered by a DD filter that is greater than $20 \%$ is considered unrealistic since a loss of $20 \%$ in the portfolio value would have led investors to withdraw their investments under normal circumstances. By the same token, having a DU of $20 \%$ would have led the portfolio to remain under-invested for an overly long period. Within the reasonable range of DD and DU, in addition to the optimal permutation, there are only 3 other permutations that outperform the unprotected MSCI World Index in terms of the Sharpe ratio: DD of -5\% and DU of 15\% that result in the Sharpe ratio of $29.07 \%$; DD of $-10 \%$ and DU of $5 \%$ that result in the Sharpe ratio of $25.76 \%$; and DD of $-15 \%$ and DU of $5 \%$ that result in the Sharpe ratio of $33.41 \%$. Thus, the minimum required DU of 5\% seems to be a sufficient indication that the portfolio has come out of its most recent trough for the MSCI World Index.

Table 1: Sharpe Ratio for the Filter Rule Strategy

The simulated Sharpe ratios are generated from different permutations of the maximum tolerable drawdown filter that triggers cash protection and the minimum required drawup filter that releases the protection for the MSCI World Index over the period from 1970 to 2008 .

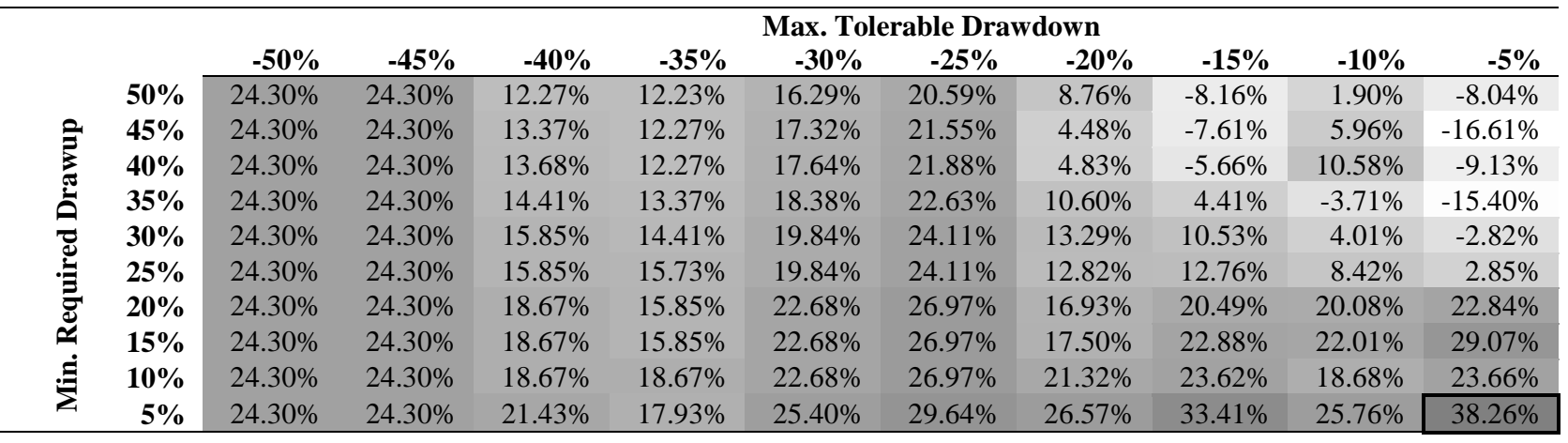

Table 2 demonstrates the Sharpe ratios of the protected MSCI World Index under the EMA strategy based on the simulated permutations of FMA and SMA. The optimal permutation that maximizes the Sharpe ratio of the EMA strategy is indicated in the bold box. Examining the heat map of Table 2 shows that the Sharpe ratio of $42.84 \%$ for the EMA strategy is achieved when the FMA and the SMA are set at $100 \%$ and $80 \%$ respectively. Since the FMA of $100 \%$ in the optimal permutation represents the cumulative total return for the MSCI World Index since inception, the SMA of $80 \%$ can be viewed as the long-term support level for the MSCI World Index when the index is above the SMA, and the long-term resistant level for the MSCI World Index when the index is below the SMA. 
For permutations that set the FMA equal to the SMA, the resulting Sharpe ratio represents the Sharpe ratio of the unprotected MSCI World Index since no signal will be generated under these circumstances. The two permutations in Table 2 that are classified in this category include the permutation that sets both the FMA and the SMA to $100 \%$ and the permutation that sets both the FMA and the SMA to $10 \%$. Using the Sharpe ratio of the unprotected MSCI World Index as the benchmark (24.30\%), there are quite a few permutations that result in outperforming Sharpe ratios based on the EMA strategy. The combination of a SMA of $20 \%$ with any FMA generally produces outperforming Sharpe ratios for the EMA strategy. It is also noted that the permutation that involves the FMA of $100 \%$ and the SMA of $90 \%$ results in a Sharpe ratio of $41.12 \%$, which is very close to the optimal Sharpe ratio of $42.84 \%$.

Table 2: Sharpe Ratio for the EMA Strategy

The simulated Sharpe ratios for the MSCI World Index are generated from different permutations of FMA and SMA crossovers over the period from 1970 to 2008. When the FMA breaks through the SMA from above, the cash protection signal is triggered until the FMA breaks through the SMA from below, which releases the cash protection.

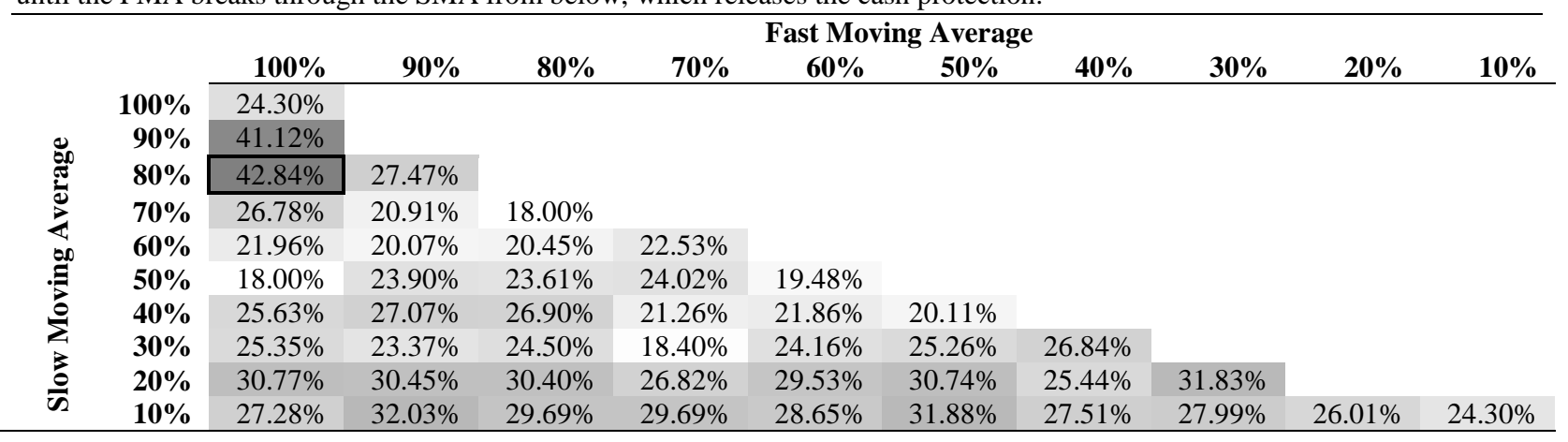

\section{Periodic Performance for the Optimal Market Timing Strategies}

The periodic log cumulative returns for the permutation of the maximum tolerable DD of $-5 \%$ and the minimum required DU of 5\% that results in the optimal Sharpe ratio for the filter rule strategy is compared to that of the unprotected MSCI World Index over the examination period from 1970 to 2008 as shown in Figure 1. The protected/hedged periods under the filter rule strategy are highlighted by vertical shadings. A relative strength index, calculated as the value of the protected index divided by the value of the unprotected index, is provided to give indications as to whether economic value is created during the protected periods.

Examining the relative strength index reveals that the filter rule strategy provides economic benefits over longer rather than shorter periods of uncertainties such as the oil crisis of 1973, the crash of the dot-com bubble in early 2000s and the global financial crisis of 2008. This can be seen from the periodic drawdowns illustrated in Figure 2 in that the unprotected index suffers from abnormally large drawdown of more than $30 \%$ during these periods. As is the case for all market timing models, the signals provided by the protection, in general, lag the actual economic events, which results in the market timing strategies being more effective for prolonged economic downturns. For shorter or temporary economic shocks, the filter rule strategy might fail to trigger timeous action due to delayed DD and DU signals. It is also noted that a premature DU signal is generated in 2003, which results in the protection being released too early. The consequence is a sharp temporary drawdown for the portfolio just before the market rebound. Although the maximum tolerable DD is set at $-5 \%$, the periodic DD in Figure 2 often exceeds this threshold due to some abnormally large downward monthly correction in the index values during these periods. This suggests that the monthly examination of DD and DU filters might not be frequent enough to provide timeous signals for the filter rule strategy. 


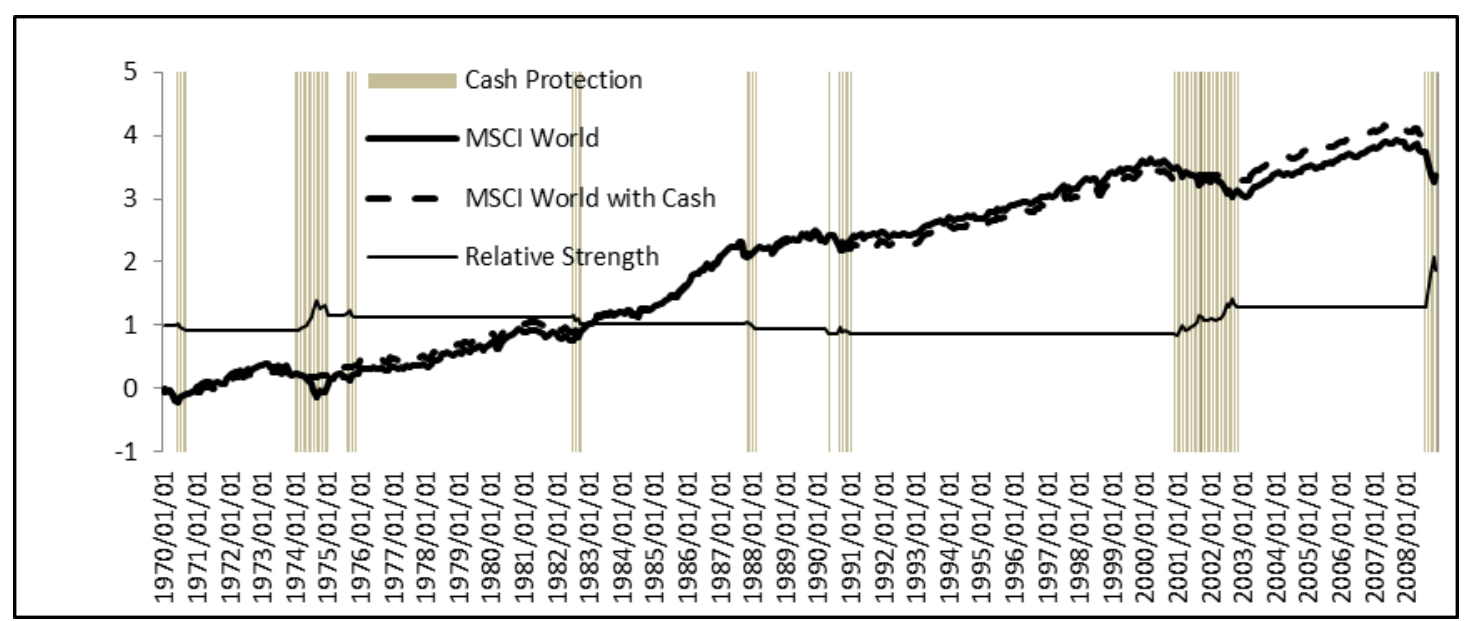

Figure 1: Log Cumulative Returns for the Optimal Filter Rule Strategy

This figure illustrates the log cumulative returns of the unprotected MSCI World Index and that of the optimal filter rule strategy that applies a maximum tolerable DD of $-5 \%$ and a minimum required DU of 5\% over the period from 1970 to 2008 . The vertical shadings represent the months in which the protection is triggered. The relative strength index, calculated as the value of the protected index divided by the value of the unprotected index, is also provided in this figure.

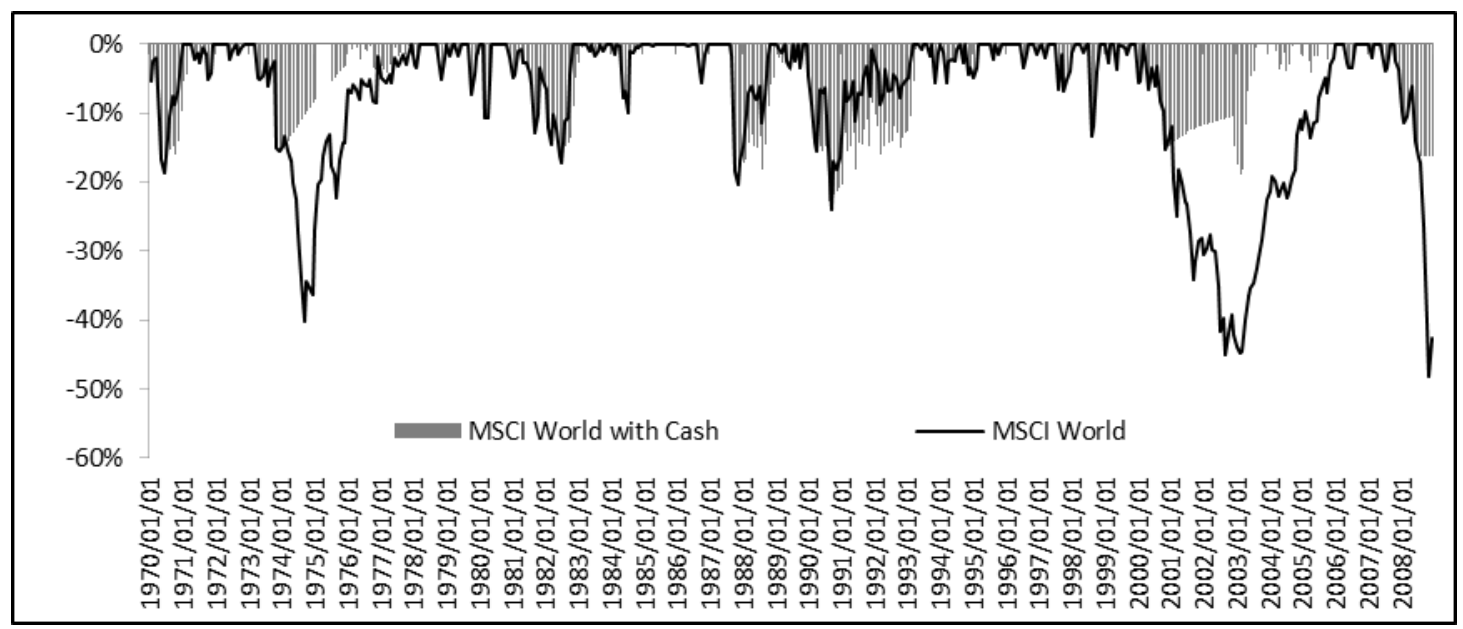

Figure 2: Periodic Drawdown for the Optimal Filter Rule Strategy

The periodic drawdown for the unprotected MSCI World Index and that of the optimal filter rule strategy that applies a maximum tolerable DD filter of $-5 \%$ and a minimum required DU filter of 5\% over the period from 1970 to 2008 are illustrated.

The periodic log cumulative returns for the permutation of the FMA of $100 \%$ and the SMA of $80 \%$ that results in the optimal Sharpe ratio for the EMA strategy over the examination period from 1970 to 2008 is illustrated in Figure 3. Similar to the findings of the optimal filter rule strategy, the optimal EMA strategy is more effective during prolonged structural economic downturns. The periodic drawdowns of the optimal EMA strategy and that of the unprotected MSCI World Index over the period from 1970 to 2008 are illustrated in Figure 4. The premature signal of economic recovery observed for the optimal filter rule strategy in 2003 is not exhibited under the optimal EMA strategy. It is also noted that the periodic DD of the optimal EMA strategy is less severe compared to the periodic DD of the optimal filter rule strategy. This suggests that the signals provided by the EMA strategy are better indications for the expected future movements of the unprotected index relative to the signals provided by the filter rule strategy. 


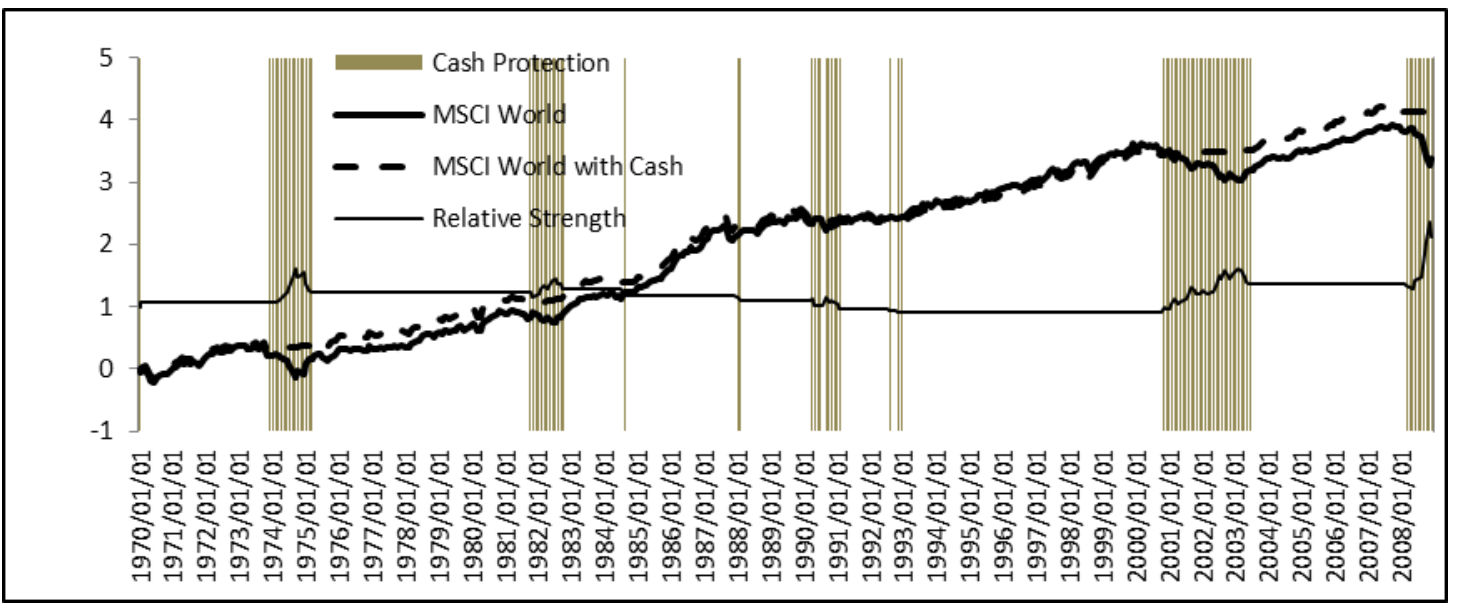

Figure 3: Log Cumulative Returns for the Optimal EMA Strategy

This figure illustrates the log cumulative returns of the unprotected MSCI World Index and that of the optimal EMA strategy that applies the FMA of $100 \%$ and the SMA of $80 \%$ over the period from 1970 to 2008 . The vertical shadings represent the months in which the protection is triggered. The relative strength index, calculated as the value of the protected index divided by the value of the unprotected index is also provided.

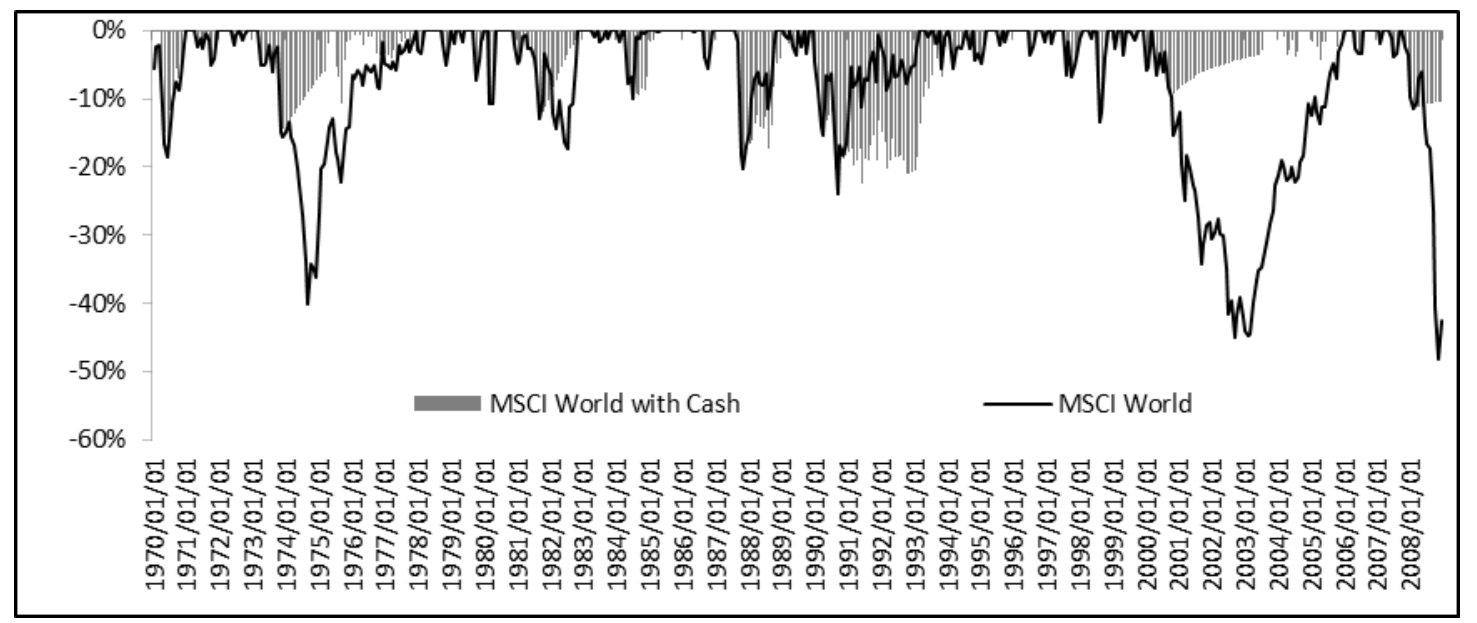

Figure 4: Periodic Drawdown for the Optimal EMA Strategy

The periodic drawdown of the unprotected MSCI World Index and that of the optimal EMA strategy that applies the FMA of $100 \%$ and the SMA of $80 \%$ over the period from 1970 to 2008 are illustrated.

\section{Summary of Performance Statistics}

The summarized performance statistics for the unprotected MSCI World Index, the fully-protected strategy $(100 \%)$ and the partially-protected strategy (50\%) are presented in Table 3. The returns for all four protected strategies are higher than the unprotected MSCI World Index with their standard deviations, VaR and maximum drawdowns less than those of the unprotected index. Comparing the performance statistics of the respective strategies that apply different levels of protection (100\% versus 50\%) reveals that the fully-protected indexes outperform the partially-protected counterparts for both the filter rule strategy and the EMA strategy. The optimal filter rule strategy has approximately $24.07 \%$ of the months in cash compared to $35.19 \%$ of the months in cash for the optimal EMA strategy over the examination period. The additional months being hedged under the optimal EMA strategy are deemed effective in that the optimal EMA strategy outperforms the filter rule strategy on a risk-adjusted basis in terms of the Sharpe ratio. This outperformance is primarily due to the additional risk reduction exhibited under the optimal EMA strategy compared to the optimal filter rule strategy. This serves as evidence that the timing 
of signals for the optimal EMA strategy is more accurate and prompt compared to the timing of signals generated by the optimal filter rule strategy.

Table 3: Performance Statistics of the Market Timing Strategies (1970 to 2008)

Using the unprotected MSCI World Index as the benchmark, the risk-return characteristics of the optimal filter rule strategy and the EMA strategy with different levels of protection are demonstrated.

\begin{tabular}{|c|c|c|c|c|c|}
\hline & \multirow{2}{*}{$\begin{array}{l}\begin{array}{l}\text { Benchmark } \\
\text { (No Protection) }\end{array} \\
\text { Unprotected } \\
\text { MSCI World }\end{array}$} & \multicolumn{2}{|c|}{$\begin{array}{l}\text { Market Timing Based on } \\
\text { Filter Rule Threshold }\end{array}$} & \multicolumn{2}{|c|}{$\begin{array}{l}\text { Market Timing Based on } \\
\text { Exponential Moving Average }\end{array}$} \\
\hline & & $\begin{array}{l}\text { MSCI World } \\
-5 \% \text { DD } \\
5 \% \text { DU } \\
100 \% \text { Hedge }\end{array}$ & $\begin{array}{l}\text { MSCI World } \\
\mathbf{5 \%} \text { DD } \\
\mathbf{5 \%} \text { DU } \\
\mathbf{5 0 \%} \text { Hedge }\end{array}$ & $\begin{array}{l}\text { MSCI World } \\
100 \% \text { FMA } \\
80 \% \text { SMA } \\
100 \% \text { Hedge }\end{array}$ & $\begin{array}{l}\text { MSCI World } \\
100 \% \text { FMA } \\
\mathbf{8 0 \%} \text { SMA } \\
50 \% \text { Hedge }\end{array}$ \\
\hline $\begin{array}{l}\text { Geometric } \\
\text { Return }\end{array}$ & $11.62 \%$ & $12.78 \%$ & $12.30 \%$ & $12.57 \%$ & $12.26 \%$ \\
\hline $\begin{array}{l}\text { Standard } \\
\text { Deviation }\end{array}$ & $15.08 \%$ & $12.60 \%$ & $13.26 \%$ & $10.77 \%$ & $12.00 \%$ \\
\hline $\begin{array}{l}\text { Sharpe } \\
\text { Ratio }\end{array}$ & $24.30 \%$ & $38.26 \%$ & $32.70 \%$ & $42.84 \%$ & $35.86 \%$ \\
\hline $5 \%$ VaR & $-5.99 \%$ & $-4.33 \%$ & $-4.52 \%$ & $-3.36 \%$ & $-4.31 \%$ \\
\hline $\begin{array}{l}\% \text { of Months } \\
\text { in Cash }\end{array}$ & $0.00 \%$ & $24.07 \%$ & $24.07 \%$ & $35.19 \%$ & $35.19 \%$ \\
\hline $\begin{array}{l}\text { Maximum } \\
\text { Drawdown }\end{array}$ & $-40.12 \%$ & $-24.30 \%$ & $-24.02 \%$ & $-16.27 \%$ & $-27.24 \%$ \\
\hline
\end{tabular}

\section{SUMMARY AND CONCLUSION}

Empirical literature suggests that market timing strategies that involve frequent switching based on shortterm forecasts generally require high predicting accuracy to justify their trading costs. Motivated by this argument, this research develops protection-based market timing strategies that target at preserving portfolio values during prolonged periods of uncertainty to mitigate the adverse impact of systemic risk. The benefits provided by avoiding structural economic shocks are often found to be larger than the gains derived from being involved in the bull market.

The two strategies developed in this study include the filter rule strategy that generates signals by observing the periodic DD and DU; and the EMA strategy that generates signals based on the crossover of the FMA and the SMA. The strategies are tested on the cumulative total U.S. dollar return of the MSCI World Index since inception in 1970 to 2008. When the maximum tolerable DD and the minimum required DU for the filter rule strategy are set at $-5 \%$ and $5 \%$ respectively, the Sharpe ratio for the protected index is maximized over the examination period. On the other hand, the FMA of $100 \%$ and the SMA of $80 \%$ maximizes the Sharpe ratio of the EMA strategy.

As is the case for all market timing models, the signals generated by both models lag the actual economic events, which results in the strategies being more effective for prolonged economic downturns. When the historical risk-return characteristics of the optimal filter rule strategy are compared and contrasted to the optimal EMA strategy, it is found that the signals generated by the optimal EMA strategy are much more accurate than the signals provided by the optimal filter rule strategy. The optimal EMA strategy achieves a Sharpe ratio of $42.84 \%$, which is much higher than the Sharpe ratio of $38.26 \%$ achieved by the optimal filter rule strategy. The outperformance of the optimal EMA strategy is mainly due to the risk reduction which resulted from timely predictions regarding peaks and troughs, resulting in the optimal EMA strategy being more resilient during major economic downturns. Suggested areas for further research include tests of the protection-based market timing strategies on style- or sectorbased portfolios in the global equity markets. 


\section{ACKNOWLEDGEMENTS}

This work is supported by the National Research Foundation (NRF) of South Africa.

\section{AUTHOR INFORMATION}

Dr. Heng-Hsing Hsieh, CFA is the Head of Finance in the School of Business and Finance at the University of the Western Cape, South Africa. He is a member of the International Institute of Forecasters (IIF) and a member of the South African Institute of Financial Markets (SAIFM). E-mail: ahsieh@uwc.ac.za (Corresponding author)

Dr. Kathleen Hodnett is currently a Research Fellow (funded by the National Research Foundation (NRF) of South Africa) and a Senior Lecturer in the School of Business and Finance at the University of the Western Cape, South Africa. She is a member of the International Institute of Forecasters (IIF) and an associate of the South African Institute of Financial Markets (SAIFM).

Prof. Paul van Rensburg is the Frank Robb Professor of Finance at the University of Cape Town, South Africa. He is the principal of Salient Quantitative Investment Management.

\section{REFERENCES}

1. Ang A and Bekaert G (2004), “How Regimes Affect Asset Allocation”, Financial Analysts Journal, vol 60, no 2, 86-99

2. Ballio M, Getmansky M and Pelizzon L (2006), Dynamic Risk Exposure of Hedge Funds: A RegimeSwitching Approach, Working Paper, 1-55

3. Ballio M, Getmansky M and Pelizzon L (2009), Crises and Hedge Fund Risk, Working Paper, 1-60

4. $\quad$ Bauer R, Dahlquist J R (2001), "Market Timing and Roulette Wheels", Association for Investment Management and Research, vol 57, no 1, 28-40

5. Bodie Z, Kane A and Marcus A J (2008), Investments, $7^{\text {th }}$ Edition, McGraw Hill

6. Calson M and Steiman J (2008), Market Conditions and Hedge Fund Survival, Finance and Economics Discussion Series, Divisions of Research and Statistics and Monetary Affairs, Federal Reserve Board, Washington, D.C

7. Estrada J (2008), "Black Swans and Market Timing: How Not to Generate Alpha”, The Journal of Investing, vol 17, no 3, 20-34

8. Faber M T (2009), “A Quantitative Approach to Tactical Asset Allocation”, The Journal of Wealth Management, 1-47

9. Chua J H, Woodward R S and To E C (1987), "Potential Gains from Stock Market Timing in Canada", Financial Analysts Journal, vol 43, no 5, 50-56

10. Clarke R G, FitzGerald M T, Berent P and Statman M (1989), "Market Timing with Imperfect Information", Financial Analysts Journal, vol 45, no 6, 27-36

11. Kester G W (1990), "Market Timing with Small versus Large-Firm Stocks: Potential Gains and Required Predictive Ability", Financial Analysts Journal, vol 45, no 5, 63-69

12. Resnick B G and Shoesmith G L (2002), "Using the Yield Curve to Time the Stock Market", Financial Analysts Journal, vol 58, no 3, 82-90

13. Sharpe W F (1975), "Likely Gains from Market Timing", Financial Analysts Journal, vol 31, no 2, 60-69

14. Shilling A G (1992), "Market Timing: Better Than a Buy-and-Hold Strategy", Financial Analysts Journal, vol 48, no 2, 46-50

15. Taleb N (2007), The Back Swan: The Impact of the Highly Improbable, Random House 
NOTES 